\title{
Clinical Characteristics and Short-Term Outcomes of Chronic Dialysis Patients Admitted for COVID-19 in Metro Manila, Philippines
}

This article was published in the following Dove Press journal: International Journal of Nephrology and Renovascular Disease

\author{
Isabelle Dominique Tomacruz (D) \\ Paolo Nikolai So (iD) \\ Renz Michael Pasilan \\ Jameel Kristine Camenforte \\ Maria Isabel Duavit \\ Division of Nephrology, Department of \\ Medicine, Philippine General Hospital, \\ Manila, Metro Manila, Philippines
}

Aim: Data published on COVID-19 in the Filipino population, particularly those with end stage kidney disease (ESKD) are still lacking.

Methods: We performed a retrospective, observational study of 68 ESKD patients admitted with COVID-19 infection at a tertiary hospital in Metro Manila, Philippines from April 1, 2020 to July 31 , 2020. We compared the clinical features, baseline laboratory data, treatment strategies and short-term outcomes between those who survived and those who died. We also determined the risk factors associated with mortality from COVID-19.

Results: Mean age was 54.5 years old, $66 \%$ were male. All patients admitted were on maintenance hemodialysis (HD). The most common presenting symptoms were dyspnea $(57 \%)$, fever $(47 \%)$ and cough $(38 \%)$. There was an equal number of patients on high flow nasal cannula (17.7\%) and invasive mechanical ventilation (17.7\%). ICU admission was required in $17.7 \%$ of the cohort. In-hospital death occurred in $25 \%$ of the patients. Admission $\mathrm{PaO}_{2} / \mathrm{FiO}_{2}(\mathrm{PF})$ ratios $(162 \pm 134$ versus $356 \pm 181 ; \mathrm{p}=0.0009)$ were lower, and procalcitonin $(6.07 \pm 10.5 \mathrm{ng} / \mathrm{mL}$ versus $0.73 \pm 3.61 \mathrm{ng} / \mathrm{mL} ; \mathrm{p}=0.02)$, lactate dehydrogenase $(396 \pm 274 \mathrm{U} / \mathrm{L}$ versus $282 \pm 148 \mathrm{U} / \mathrm{L} ; \mathrm{p}=0.03)$, and white blood cell counts $\left(10 \pm 7.3 \times 10^{9} / \mathrm{L}\right.$ versus $6.3 \pm$ $\left.4.2 \times 10^{9} / \mathrm{L} ; \mathrm{p}=0.0039\right)$ were significantly higher among those who died compared to those who survived. After adjusting for confounders, only low PF ratio (HR 1.01 for every unit decrease, 95\% CI 1-1.01) and need for ventilation (HR 6.45, 95\% CI 1.16-35.97) conferred a significant risk for in-hospital mortality.

Conclusion: Short-term, in-hospital mortality is high among patients on chronic hemodialysis admitted for COVID-19 infection. They present similarly with the general population. Low PF ratio on admission and need for ventilation are independent risk factors for inhospital mortality.

Keywords: coronavirus, COVID-19, hemodialysis, dialysis, kidney failure

\section{Introduction}

Since its emergence in China, the novel coronavirus (COVID-19) caused by the severe acute respiratory syndrome coronavirus 2 (SARS-CoV-2) has rapidly become a global pandemic. ${ }^{1,2}$ It presents a unique challenge to patients with ESKD on kidney replacement therapy. As the disease spreads in the community, many of these patients continue to require thrice-weekly dialysis treatments and are unable to avoid travelling during this time. Continuous dialysis treatments in outpatient dialysis centers pose the risk of spread of infection among patients and healthcare staff since these closed spaces may cause easy transmission. ${ }^{3,4}$ In the United Kingdom, a high seroprevalence $(36.2 \%)$ of
Correspondence: Isabelle Dominique Tomacruz

Division of Nephrology, Department of Medicine, Philippine General Hospital,

Taft Avenue, Manila, Metro Manila, I000, Philippines

Email ivtomacruz@up.edu.ph
International Journal of Nephrology and Renovascular Disease 2021:|4 4|-5|

41

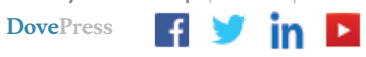

http: $/$ doi.org/10.2147111NRD.5287455 
SARS-CoV-2 antibodies was observed among patients receiving in-center HD. ${ }^{5}$ With implementation of lockdown measures, a decrease in total number of dialysis patients infected with COVID-19, from 2517 to 2404, was seen in 19 major hospitals in India. Outpatient consults were reduced by $92.3 \%$, while inpatient service decreased by $61 \% .{ }^{6}$ Given the high rate of infectivity of COVID-19, the challenge of "social distancing" and the increased risk of mortality among elderly and those with comorbidities such as chronic kidney disease, it seems prudent to pay particular attention to this vulnerable population.

The Philippines is the second-most populated country in the Association of Southeast Asian Nations (ASEAN) region, home to over 100 million people. Although exact data is lacking, a steep rise in the prevalence of chronic kidney disease has been reported, with a $400 \%$ increase in the number of patients on some form of kidney replacement therapy over the last 10 years. ${ }^{7}$ As of October 2020 , the Philippines ranks second in the ASEAN, with the most number of COVID-19 cases with 470,650 confirmed cases and 9124 total deaths to date. ${ }^{2}$ The first case of COVID-19 was reported in January 2020, and local transmission was documented in March 2020. Since then, its capital and one of the largest metropolitan cities, Metro Manila, continues to be at epicenter of this pandemic in the Philippines. ${ }^{8}$

The Philippine General Hospital (PGH) is one of first three hospitals in Metro Manila designated by the Department of Health as a referral center for moderate and severe cases of COVID-19. ${ }^{9}$ As of December 2020, more than 2400 patients have been hospitalized at PGH for COVID-19 infection. The PGH Nephrology Division provides care to over 400 patients with ESKD on chronic HD from all over the country every year. Studies describing local experience are currently limited; hence, this study aims to determine the clinical profiles and short-term outcomes of chronic dialysis patients admitted for COVID-19 in Metro Manila, Philippines during the first few months of the pandemic.

\section{Methods}

\section{Study Design and Participants}

This is a single-center, retrospective, observational study conducted at the PGH from April 1, 2020 to July 31, 2020. Data included all patients 18 years old and above, undergoing maintenance peritoneal dialysis (PD) or HD, hospitalized for COVID-19 disease confirmed by at least one positive result for SARS-CoV-2 on real-time polymerase chain reaction (RT-PCR) testing of nasopharyngeal samples. Kidney transplant recipients and patients who expired or were discharged prior to completion of baseline imaging and laboratory tests were excluded. This study was approved by the University of the Philippines-Manila Research Ethics Board. All procedures performed involving human participants were in accordance with the ethical standards of the institutional research committee and with the 1964 Declaration of Helsinki and its later amendments. Informed consent was waived since the investigators analyzed anonymized data.

In early March 2020, when COVID-19 cases were rapidly increasing in the city, the PGH HD Unit shifted to bedside HD for all infected COVID-19 patients needing kidney replacement therapy so as to decrease transmission of infection within the hospital. Personal protective equipment was provided by the hospital to all healthcare workers entering the designated wards for COVID-19 patients. We continued to provide dialysis care six days a week, but remained on-call every Sunday for emergency HD should the need arise. HD nurse to patient ratio in these areas was $1: 3$. The primary modality used was conventional, intermittent HD with either low-flux or high-flux dialyzers. These included the BBraun Diacap LOPS 18, Nipro Elisio-19L low-flux dialyzers and BBraun Diacap HIPS 18, Nipro Elisio-17H, and Nipro Elisio-19H high-flux dialyzers. Dialysis cartridges were on a strict single-use basis only and were discarded after every procedure. Dialysis was decreased to twice-weekly baseline, using high-flux dialyzer membranes (unless with contraindications), at the shortest time possible but not less than 2 hours and blood flow rates were adjusted accordingly. Patients were assessed by a nephrologist-on-duty on a daily basis to determine if additional dialysis was warranted.

Only one nephrologist per week was assigned to see and attend to all patients needing dialysis. Interviews via telephone calls were maximized where possible, and most orders were done via our electronic health records system so as to minimize exposure.

Admissions and referrals for PD continued; however, lack of available PD fluids as well as limited automated cyclers for automated PD were some logistical challenges.

\section{Data Collection and Definition of Variables}

Data was collected through chart review of both paper and electronic medical records. This included demographic 
information on patients' age, sex, dialysis vintage, cause of kidney failure, and comorbidities. Presenting symptoms, baseline laboratory results on admission, chest X-ray findings, ventilatory support, treatment measures, need for ICU admission, and length of hospital stay were also obtained.

Comorbidities include hypertension, cardiovascular disease, diabetes mellitus, chronic lung disease and presence of malignancy. History of hypertension was determined by history of intake or oral antihypertensive agents and/or with records of blood pressure monitoring $\geq 140 / 90$ $\mathrm{mmHg}$ on more than 2 occasions. History of diabetes mellitus was determined by fasting blood sugar $\geq 126 \mathrm{mg} / \mathrm{dL}$ or HBa1c $\geq 6.5 \%$ or if by history was being treated by oral hypoglycemic agents and/or insulin formulations. Cardiovascular disease was determined by history of ischemic heart disease and/or by evidence of coronary artery disease by cardiac catheterization reports, history of percutaneous coronary intervention or coronary artery bypass graft surgery. History of heart failure by history, clinical symptoms, or by evidence on 2D echocardiography was also included. Chronic obstructive pulmonary disease or chronic lung disease was determined by history and/or was confirmed by a previous spirometry report. Malignancy was defined as any evidence of active malignancy in any organ either by history or documentation in medical records.

The definition of Chronic Kidney Disease (CKD) is based on the 2012 Kidney Disease: Improving Global Outcomes guideline: abnormalities in kidney structure of function, present for $>3$ months. Specifically, CKD stage $5 \mathrm{D}$ or "kidney failure with kidney replacement therapy" is the presence of GFR $<15 \mathrm{~mL} / \mathrm{min}$ per $1.73 \mathrm{~m}^{2}$ with treatment by either PD, HD, or kidney transplantation; however, kidney transplant recipients were excluded in our study. The recorded causes of kidney failure include common causes documented in our country namely: hypertension, diabetes mellitus, chronic glomerulonephritis, or obstructive uropathy, as documented in medical records.

Baseline laboratory results were defined as the first data collected within 24 hours of admission and included hemoglobin concentration, lymphocyte count, platelet count, aspartate aminotransferase, alanine aminotransferase, and inflammatory markers: ferritin, c-reactive protein (CRP), procalcitonin, and lactate dehydrogenase (LDH) concentration. The presence of chest X-ray abnormalities were classified in three: unilateral, bilateral or diffuse pulmonary infiltrates as analyzed by a trained radiologist and recorded or documented in the medical charts.

Treatment measures included antiviral therapy, use of azithromycin, chloroquine or hydroxychloroquine, corticosteroid therapy, use of IL-6 receptor inhibitor tocilizumab, convalescent plasma, or hemoperfusion therapy.

Outcomes measured were (a) need for noninvasive ventilator support namely high flow nasal cannula, or invasive mechanical ventilation, (b) need for ICU admission, (c) length of hospital stay, and (d) final patient outcomes of death, or recovery.

\section{Statistical Analysis}

Descriptive statistics were used in the analysis of this study. Continuous variables are expressed as median with interquartile range (IQR) as appropriate. Frequency and percentage are used to describe categorical variables, proportion of patients who will require intensive care unit (ICU) admission and the proportion of patients who have recovered or expired.

Baseline characteristics, clinical and radiologic findings between survivors and mortalities were compared using Student's $t$-test or Mann-Whitney $U$-test for quantitative variables, and chi-square test or Fisher exact test for qualitative variables, as appropriate. Box-and-whiskers graphs were constructed for factors with significant differences when grouped according to survival.

Univariate logistic regression model was created to determine the risk factors for mortality from COVID-19 in our study population. Variables included age, sex, comorbidities (hypertension and diabetes mellitus), dialysis vintage, hemoglobin level, white blood count, absolute lymphocyte count, platelet count, inflammatory markers (procalcitonin, ferritin, CRP, and $\mathrm{LDH}$ ) and ventilatory support. Variables with $\mathrm{p}$ values $<0.05$ in the univariate model were included in the final multivariable logistic regression analysis.

Survival analysis was done using length of hospital stay in days as time function and mortality as failure function in analysis of interventions received during admission. Analysis was right-censored, ie, for survivors, the length of hospital stay was determined as a function of discharge/last known surviving day rather than mortality. Log rank test for equality of survival functions was done to interventions which have differences in survivor functions. Kaplan-Meier curves were constructed for interventions with significant survivor function differences. We conducted all analyses using Stata version 15.1. 


\section{Results}

Baseline characteristics of the cohort are shown in Table 1.

We identified 68 patients with ESKD on dialysis admitted with COVID-19 at the PGH from April 1 to July 31, 2020. The mean age was 54.5 years, with a higher prevalence being male $(66 \%)$. The most common comorbidities were hypertension (74.6\% of survivors and $25.5 \%$ of non-survivors) and diabetes (74.1\% of survivors and $25.9 \%$ of non-survivors). It followed that the cause of ESKD among these patients were also hypertension and diabetes. All patients were on maintenance HD, while none were on PD. The median dialysis vintage was 2 years. There were no statistically significant differences in clinical and demographic characteristics between those who survived and those who died.

\section{Clinical Characteristics and Laboratory Findings of COVID-I9 Among ESKD Patients}

The most common presenting symptoms were dyspnea (57\%), followed by fever (47\%) and cough (38\%). Other less commonly reported symptoms noted were fatigue (6\%), myalgia (3\%), and diarrhea (3\%) (Table 2). Majority (84\%) of initial chest radiographs showed bilateral opacities while only eleven patients (16\%) were noted to have unilateral infiltrates on imaging. None of the patients presented with diffuse pulmonary infiltrates on admission. There was no evidence of association between presenting symptoms or chest X-ray findings and survival.

Table I Baseline Characteristics of ESKD Patients on Dialysis Admitted for COVID-19 Infection

\begin{tabular}{|c|c|c|c|c|}
\hline Characteristics & Total $(n=68)$ & Survivors $(n=5 I)$ & Non-Survivors $(n=17)$ & p-value \\
\hline Mean Age, year (min - max) & $54.5(19-86)$ & $53.6(19-86)$ & $57.1(35-79)$ & 0.3851 \\
\hline Sex, n (\%) & & & & 0.183 \\
\hline Male & 45 & $36(80.00 \%)$ & $9(20.00 \%)$ & \\
\hline Female & 23 & $15(65.22 \%)$ & $8(34.78 \%)$ & \\
\hline \multicolumn{5}{|l|}{ Comorbidities, n (\%) } \\
\hline Hypertension & 55 & $4 \mathrm{l}(74.55 \%)$ & $14(25.45 \%)$ & 1.000 \\
\hline Diabetes mellitus & 27 & 20 (74.07\%) & 7 (25.93\%) & 1.000 \\
\hline Cardiovascular disease & I & I (I00.0\%) & - & 1.000 \\
\hline Malignancy & I & I (I00.0\%) & - & 1.000 \\
\hline Chronic lung disease & $\mathrm{I}$ & $\mathrm{I}(100.0 \%)$ & - & 1.000 \\
\hline \multicolumn{5}{|l|}{ Primary cause of ESKD, $n(\%)$} \\
\hline Hypertension & 39 & $26(66.67 \%)$ & $13(33.33 \%)$ & 0.066 \\
\hline Diabetes & 23 & $19(82.61 \%)$ & $4(17.39 \%)$ & 0.300 \\
\hline CGN & 10 & $9(90.00 \%)$ & $\mathrm{I}(10.00 \%)$ & 0.431 \\
\hline OU & 4 & $4(100.0 \%)$ & - & 0.565 \\
\hline \multicolumn{5}{|l|}{ Maintenance dialysis Modality, $\mathrm{n}(\%)$} \\
\hline Hemodialysis & 68 & $53(77.9 \%)$ & $15(22.0 \%)$ & \\
\hline Peritoneal dialysis & 0 & - & - & \\
\hline Median dialysis vintage in years (range) & $2(0.02-12)$ & $2(0.02-12)$ & $2(0.08-3)$ & 0.2594 \\
\hline
\end{tabular}


Table 2 Clinical Characteristics and Laboratory Findings of COVID-19 Among ESKD Patients on Dialysis

\begin{tabular}{|c|c|c|c|c|}
\hline Characteristics & Total $(n=68)$ & Survivors $(n=5 I)$ & Non-Survivors $(n=\mid 7)$ & p-value \\
\hline \multicolumn{5}{|l|}{ Presenting symptoms, n (\%) } \\
\hline Fever & $32(47.06 \%)$ & $26(81.25 \%)$ & $6(18.75 \%)$ & 0.262 \\
\hline Cough & $26(38.24 \%)$ & 19 (73.08\%) & 7 (26.92\%) & 0.773 \\
\hline Dyspnea & 39 (57.35\%) & 30 (76.92\%) & $9(23.08 \%)$ & 0.671 \\
\hline Rhinorrhea & - & - & - & - \\
\hline Sore throat & I (I.47\%) & I (I00.0\%) & - & 1.000 \\
\hline Myalgia & $2(2.94 \%)$ & I (50.00\%) & I (50.00\%) & 0.440 \\
\hline Fatigue & $4(5.88 \%)$ & $4(100.0 \%)$ & - & 0.565 \\
\hline Headache & I (I.47\%) & I (I00.0\%) & - & 1.000 \\
\hline Anosmia & - & - & - & - \\
\hline Diarrhea & $2(2.94 \%)$ & $2(100.0 \%)$ & - & 1.000 \\
\hline Nausea/Vomiting & I (I.47\%) & I (I00.0\%) & - & 1.000 \\
\hline \multicolumn{5}{|c|}{ Laboratory findings, median \pm IQR } \\
\hline Hemoglobin concentration, (g/L) & $94 \pm 29.5$ & $94 \pm 34$ & $95 \pm 25$ & 0.9098 \\
\hline WBC count $\left(10^{9} / \mathrm{L}\right)$ & $6.7 \pm 4.85$ & $6.3 \pm 4.2$ & $10 \pm 7.3$ & 0.0039 \\
\hline Absolute lymphocyte count & $678 \pm 871.5$ & $728 \pm 858$ & $600 \pm 934$ & 0.5567 \\
\hline Platelet count $\left(10^{9} / \mathrm{L}\right)$ & $208.5 \pm 122.5$ & $209 \pm 136$ & $208 \pm 87$ & 0.8595 \\
\hline AST (U/L) & $33.5 \pm 17.665$ & $33 \pm 18$ & $36 \pm 30$ & 0.4526 \\
\hline ALT (IU/L) & $23 \pm 21$ & $21 \pm 26$ & $26 \pm 12$ & 0.2847 \\
\hline Procalcitonin (ng/mL) & $1.52 \pm 7.04$ & $0.73 \pm 3.61$ & $6.07 \pm 10.5$ & 0.0217 \\
\hline Ferritin $(\mathrm{ng} / \mathrm{mL})$ & $954 \pm 1856$ & $882 \pm 1873$ & $1160 \pm 2325$ & 0.5472 \\
\hline $\mathrm{LDH}(\mathrm{U} / \mathrm{L})$ & $294 \pm 200$ & $282 \pm 148$ & $396 \pm 274$ & 0.0276 \\
\hline CRP (mg/L) & $57.82 \pm 103.47$ & $60.46 \pm 119.44$ & $55.41 \pm 87.53$ & 0.7921 \\
\hline PF Ratio & $326.5 \pm 239.5$ & $356 \pm 181$ & $162 \pm 134$ & 0.0009 \\
\hline Chest $X$-ray findings, $n$ (\%) & & & & 0.718 \\
\hline Unilateral & II (16.18\%) & 9 (8I.82\%) & $2(18.18 \%)$ & \\
\hline Bilateral & 57 (83.82\%) & $42(73.68 \%)$ & I5 (26.32\%) & \\
\hline Diffuse & - & - & - & \\
\hline
\end{tabular}

Data from laboratory tests on admission showed a median hemoglobin concentration of $94 \pm 29.5 \mathrm{~g} / \mathrm{L}$, with normal median white blood cell $\left(6.7 \pm 4.85 \times 10^{9} / \mathrm{L}\right)$ and platelet $\left(208.5 \pm 122.5 \times 10^{9} / \mathrm{L}\right)$ counts. Liver function tests were normal on admission (median AST and ALT
$33.5 \mathrm{U} / \mathrm{L}$ and $23 \mathrm{U} / \mathrm{L}$, respectively). Lymphopenia was noted with a median absolute lymphocyte count of $678 \pm$ 871.5 .

Statistically significant differences in admission PF ratio and some inflammatory markers were seen between 
the survivors and non-survivors. Those who died had lower $\mathrm{PF}$ ratios (162 versus $356 ; \mathrm{p}=0.0009$, Figure $1 \mathrm{~A})$, higher procalcitonin $(6.07 \mathrm{ng} / \mathrm{mL}$ versus $0.73 \mathrm{ng} / \mathrm{mL}$; $\mathrm{p}=0.02$; Figure 1B), higher $\mathrm{LDH}(396 \mathrm{U} / \mathrm{L}$ versus $282 \mathrm{U} /$ $\mathrm{L} ; \mathrm{p}=0.03$; Figure $1 \mathrm{C}$ ), and higher white blood cell counts $\left(10 \times 10^{9} / \mathrm{L}\right.$ versus $6.3 \times 10^{9} / \mathrm{L} ; \mathrm{p}=0.0039$; Figure $\left.1 \mathrm{D}\right)$ on admission, compared to those who survived. There was a trend toward lower absolute lymphocyte count (600 versus 728), and higher ferritin (1160 versus $882 \mathrm{ng} / \mathrm{mL}$ ) among those who died, but these did not reach statistical significance.

\section{Treatment Interventions Given for COVID-19}

Table 3 outlines the various therapeutic interventions given for ESKD patients with COVID-19 throughout the study period. During the course of the admission, a total of

A

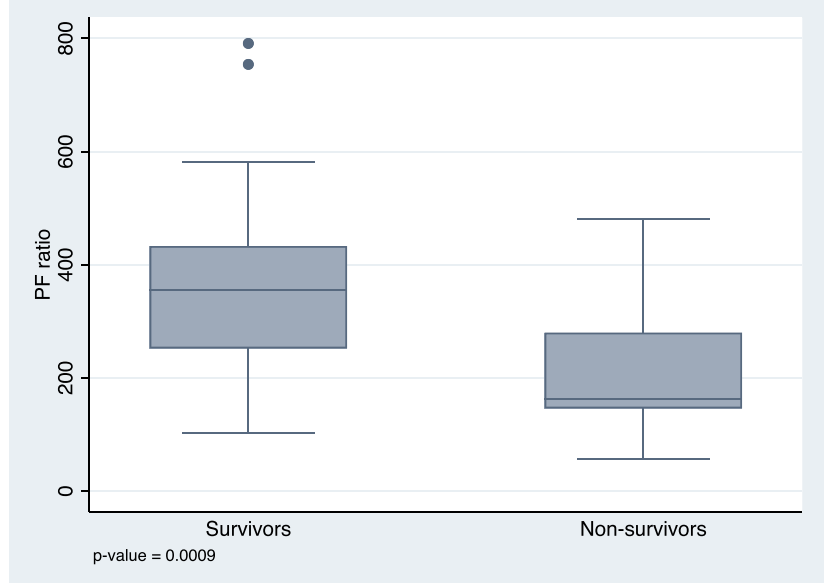

C

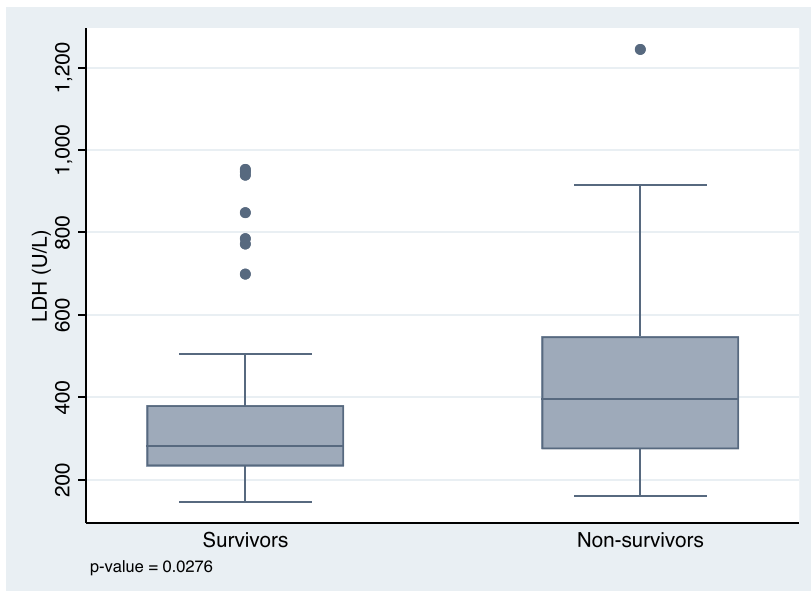

$46(67.7 \%)$ ESKD patients received azithromycin, 1 (1.5\%) received antivirals (lopinavir/ritonavir), 8 (11.76\%) received hydroxychloroquine or chloroquine, 6 $(8.82 \%)$ received tocilizumab, $6(8.82 \%)$ received corticosteroids, and $1(1.47 \%)$ received convalescent plasma therapy. Due to logistics concerns at the time, all patients remained on intermittent HD regardless of the severity of disease. Extracorporeal blood purification modality, particularly hemoperfusion, was added as adjunct therapy in 6 (8.8\%) of the ESKD patients. No significant differences were found with the use of any drug, HD modality, or hemoperfusion among survivors and non-survivors.

A significant difference in survivor function was observed when modality of ventilatory support was analyzed. There was an equal number of patients who needed noninvasive ventilation using high flow nasal cannula $(17.65 \%)$ and invasive mechanical ventilation (17.65\%); however,

B

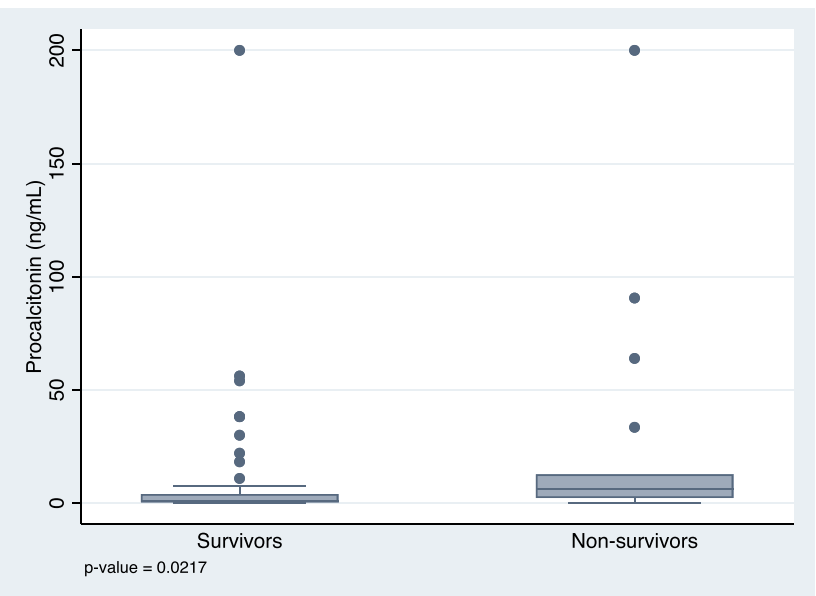

D

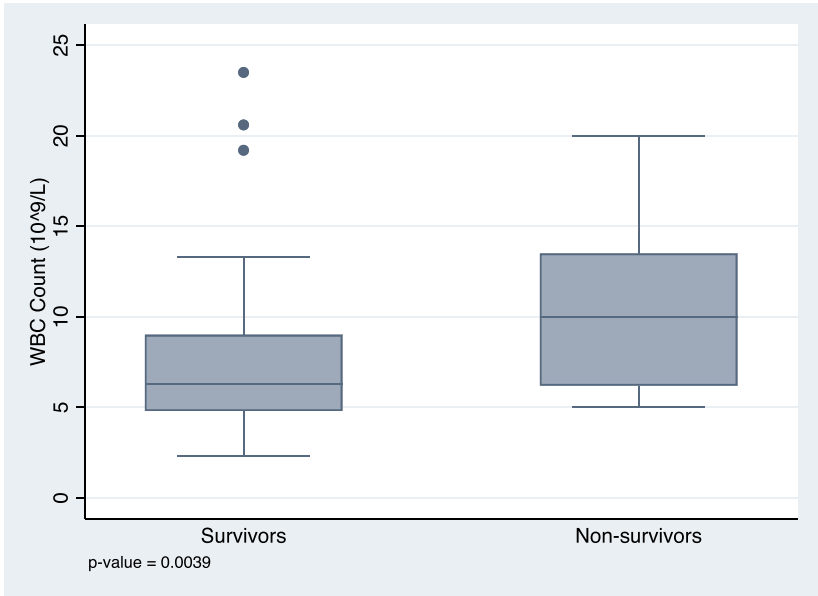

Figure I Differences in (A) PF ratio, (B) procalcitonin (ng/mL), (C) LDH (U/L), and (D) white blood cell counts $\left(\times 10^{9} / \mathrm{L}\right)$ on admission among those who survived and died; median with interquartile range. 
Table 3 Interventions Used for COVID-19 Among ESKD Patients on Dialysis

\begin{tabular}{|c|c|c|c|c|}
\hline Treatment & Total $(n=68)$ & Survivors $(n=5 I)$ & Non-Survivors $(n=\mid 7)$ & p-value \\
\hline \multicolumn{5}{|l|}{ Drugs, n(\%) } \\
\hline Azithromycin & $46(67.65 \%)$ & 37 (80.43\%) & $9(19.57 \%)$ & 0.1024 \\
\hline Antiviral (Lopinavir/Ritonavir) & I (I.47\%) & $\mathrm{I}(100.0 \%)$ & - & 0.6707 \\
\hline Chloroquine/Hydroxychloroquine & 8 (II.76\%) & $5(62.50 \%)$ & $3(37.50 \%)$ & 0.5436 \\
\hline Tocilizumab & $6(8.82 \%)$ & $4(66.67 \%)$ & $2(33.33 \%)$ & 0.5864 \\
\hline Corticosteroids & $6(8.82 \%)$ & $5(83.33 \%)$ & I (16.67\%) & 0.7412 \\
\hline Convalescent plasma & I (I.47\%) & - & I (100.0\%) & 0.0046 \\
\hline Type of ventilatory support, $n$ (\%) & & & & $<0.0001$ \\
\hline NIV & $12(17.65 \%)$ & $11(91.67 \%)$ & I (8.33\%) & \\
\hline MV & $12(17.65 \%)$ & $3(25.00 \%)$ & $9(75.00 \%)$ & \\
\hline None & $43(63.24 \%)$ & 37 (86.05\%) & $6(13.95 \%)$ & \\
\hline \multicolumn{5}{|c|}{ Hemodialysis modality on admission, $n$ (\%) } \\
\hline Intermittent HD & $68(100 \%)$ & & & \\
\hline Prolonged intermittent $\mathrm{HD}$ & 0 & - & - & \\
\hline CRRT & 0 & - & - & \\
\hline \multicolumn{5}{|l|}{ Extracorporeal therapy, n (\%) } \\
\hline Hemoperfusion & 6 & $3(50.00 \%)$ & $3(50.00 \%)$ & 0.0487 \\
\hline
\end{tabular}

a greater number of patients on invasive ventilator support died compared to those on high flow nasal cannula. KaplanMeir analysis supports these findings and showed a median survival time of 8 days on mechanical ventilation (Figure 2).

\section{Short-Term Outcomes}

By the end of the study period, in-hospital death occurred in $25 \%$ of the patients. The mean hospital length of stay was 27.5 days, with a minimum hospital stay of 2 days to a maximum of 82 days. Twelve patients (17.65\%) needed ICU admission throughout their hospital course (Table 4). These findings are supplemented by the Kaplan-Meir analysis which shows that overall, about $70 \%$ of end-stage kidney disease patients on HD admitted for COVID-19 survived and most of the mortalities occurred within 13 days from admission (Figure 3).

\section{Risk Factors for Mortality from COVID-19}

Among the risk factors assessed for mortality from COVID-19 in our study cohort, only PF ratio (HR 0.99,
95\% CI 0.99-1) and need for invasive mechanical ventilation (HR 6.45 95\% CI 1.16-35.97) conferred significant risk for in-hospital mortality (Table 5). After adjusting for confounders, multivariate analysis showed that every unit decrease in PF ratio was associated with a $1 \%$ increase in mortality, and those on mechanical ventilation had a sixfold increase in mortality compared to those not on ventilatory support.

\section{Discussion}

Data published on COVID-19 in the Filipino population, particularly those with ESKD are still lacking. Our study aims to describe the clinical features, laboratory data, treatment strategies, and short-term outcomes of 68 ESKD patients on maintenance kidney replacement therapy requiring admission for COVID-19 in a large government-run urban hospital in Metro Manila, Philippines.

In our patient cohort, those with ESKD admitted for COVID-19 were found to be older, with a greater number of comorbidities compared to the general population. A retrospective cohort study of adult inpatients diagnosed 


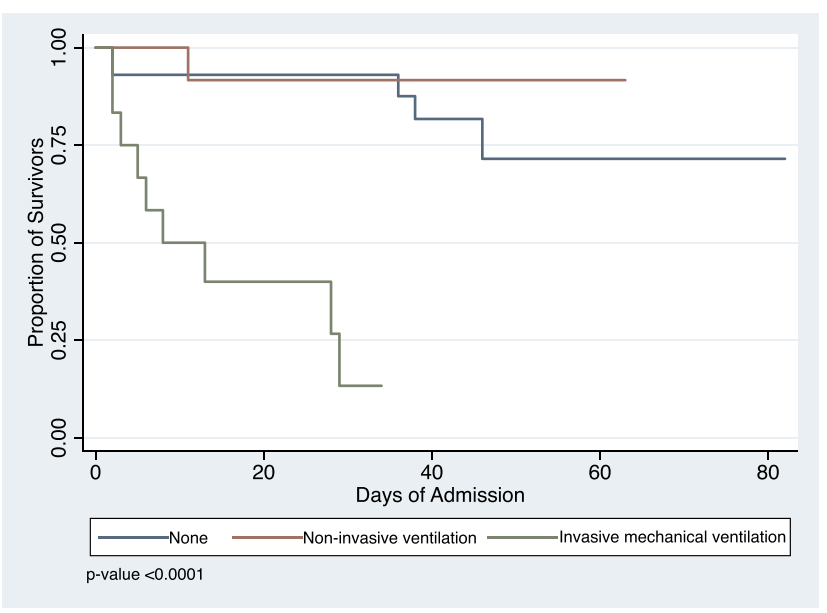

Figure 2 Kaplan-Meier analysis of survival according to type of ventilatory support among ESKD patients on HD admitted for COVID-19.

with COVID-19 in two hospitals in Wuhan, China early in the pandemic reported older age, as one of the risk factors associated with increased odds of death. ${ }^{10}$ Hypertension, diabetes, cardiovascular disease, chronic kidney disease, chronic lung disease, cancer and obesity have also been found to be associated with disease severity. ${ }^{10-13}$ We know that age and comorbidity are also strongly associated with ESKD. Given these risk factors, along with the inability to practice general social distancing measures due to the need for continuous dialysis, patients with ESKD continue to be a vulnerable population prone to infection of increased severity.

There are several controversies regarding the clinical course of COVID-19 in ESKD patients. Early in the pandemic, it was thought that they may present with mild, atypical symptoms. An observational study from a single center in Wuhan described only $11 \%$ presenting with fever, $8 \%$ with fatigue, $3 \%$ with symptoms of cough, chest pain and nausea, while the rest did not present with any obvious symptoms. ${ }^{14}$ A study on HD patients with COVID-19 in Mumbai, India reported one-third of patients

Table 4 Short-Term Outcomes of ESKD Patients on Hemodialysis Admitted for COVID-19

\begin{tabular}{|l|l|}
\hline $\begin{array}{l}\text { Need for ICU admission, } \\
\mathrm{N}(\%)\end{array}$ & $12(17.65 \%)$ \\
\hline $\begin{array}{l}\text { Mean length of hospital stay in days, median } \\
(\mathrm{min}-\mathrm{max})\end{array}$ & $27.5(2-82)$ \\
\hline Died in hospital, $\mathrm{n}(\%)$ & $17(25.00 \%)$ \\
\hline Recovered, $\mathrm{n}(\%)$ & $51(75.00 \%)$ \\
\hline
\end{tabular}

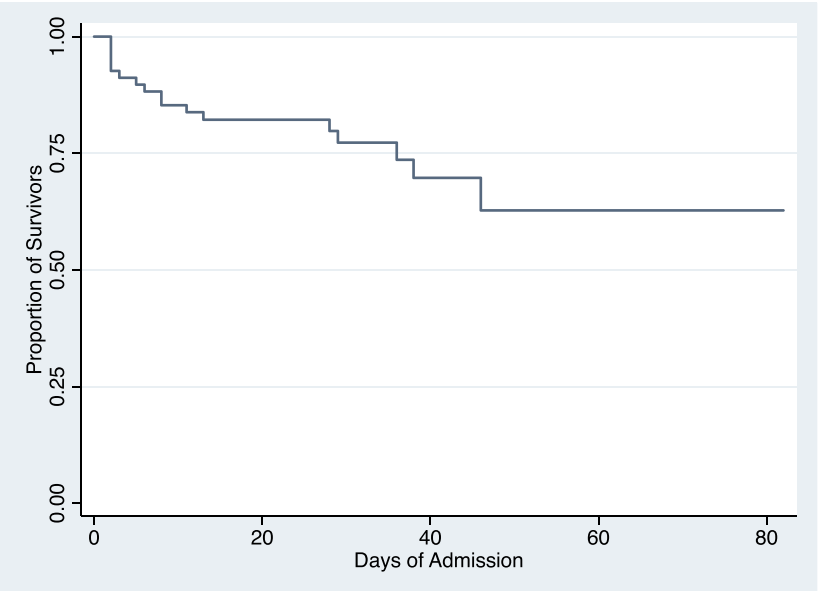

Figure 3 Kaplan-Meier analysis of survival among end-stage kidney disease patients on hemodialysis admitted for COVID-19.

to be asymptomatic with up to $50 \%$ of patients presenting with either none or only mild symptoms of COVID-19. ${ }^{15}$ Another report from California described a man on maintenance HD who was admitted for severe pulmonary disease from COVID-19 but had initially presented with diarrhea, nausea, vomiting and fever. ${ }^{16}$ In contrast to these reports, our patient cohort presented with symptoms similar to the general population (57\% with dyspnea, $47 \%$ with fever and $38 \%$ with cough). Clinical presentations were not significantly different among those who died and those who survived.

Second, an early observational study from Wuhan hypothesized that patients with ESKD are unable to mount a robust immune response that may lead to the socalled cytokine storm associated with increased disease severity and mortality. An analysis of blood samples from $230 \mathrm{HD}$ patients showed a remarkable reduction in the numbers of $\mathrm{T}$ cells, $\mathrm{T}$ helper cells, killer $\mathrm{T}$ cells, NK cells, as well as lower serum levels of inflammatory cytokines, compared to non-HD patients with COVID-19. It was observed that most of these patients experienced mild disease and none were admitted in the ICU. ${ }^{14}$ On the contrary, our study demonstrates that there was a trend towards higher $\mathrm{LDH}$, procalcitonin, WBC counts and lower PF ratios among ESKD patients who died compared to those who survived. These findings are consistent with later studies that demonstrate that elevated inflammatory makers on admission may be associated with increased disease severity and worse outcomes regardless of the intrinsic immunosuppression seen in ESKD. ${ }^{17,18}$

Treatment strategies also varied throughout the duration of this study. From March to May 2020, the use of 
Table 5 Risk Factors for In-Hospital Mortality Among ESKD Patients on Hemodialysis Admitted for COVID-19

\begin{tabular}{|c|c|c|c|c|c|c|}
\hline \multirow[t]{2}{*}{ Factors } & \multicolumn{3}{|c|}{ Univariable } & \multicolumn{3}{|c|}{ Multivariable } \\
\hline & HR & $95 \% \mathrm{Cl}$ & p-value & HR & $95 \% \mathrm{Cl}$ & $p$-value \\
\hline Age & 1.02 & $0.98-1.05$ & 0.421 & & & \\
\hline \multicolumn{7}{|l|}{ Sex } \\
\hline Male & Reference & & & Reference & & \\
\hline Female & 2.07 & $0.78-5.45$ & 0.142 & & & \\
\hline \multicolumn{7}{|l|}{ Comorbidities } \\
\hline Hypertension & 1.00 & $0.29-3.50$ & 0.996 & & & \\
\hline Diabetes mellitus & 1.20 & $0.45-3.16$ & 0.718 & & & \\
\hline Dialysis vintage & 0.75 & $0.53-1.05$ & 0.095 & & & \\
\hline \multicolumn{7}{|l|}{ Laboratory findings } \\
\hline Hemoglobin & 1.01 & $0.98-1.03$ & 0.707 & & & \\
\hline WBC count & 1.12 & $1.04-1.21$ & 0.003 & 1.00 & $0.87-1.15$ & 0.999 \\
\hline Absolute lymphocyte count & 1.00 & $1.00-1.00$ & 0.849 & & & \\
\hline Platelet count & 1.00 & $1.00-1.00$ & 0.692 & & & \\
\hline Procalcitonin & 1.01 & $1.00-1.02$ & 0.034 & 1.01 & $1.00-1.03$ & 0.064 \\
\hline Ferritin & 1.00 & $1.00-1.00$ & 0.554 & & & \\
\hline $\mathrm{LDH}$ & 1.00 & $1.00-1.00$ & 0.020 & 1.00 & $1.00-1.00$ & 0.327 \\
\hline hsCRP & 1.00 & $1.00-1.00$ & $0.74 I$ & & & \\
\hline PF ratio & 0.99 & $0.99-1.00$ & $<0.001$ & 0.99 & $0.99-1.00$ & 0.025 \\
\hline \multicolumn{7}{|l|}{ Ventilatory support } \\
\hline NIV & 0.61 & $0.07-5.06$ & 0.646 & & & \\
\hline MV & 13.65 & $3.96-47.09$ & $<0.001$ & 6.45 & $1.16-35.97$ & 0.034 \\
\hline
\end{tabular}

chloroquine and hydroxychloroquine, azithromycin, as well as the antiviral (Lopinavir/Ritonavir) were included in the investigational therapies used by our infectious disease specialists. By June, the use of these medications was stopped, and the interleukin-6 inhibitor, Tocilizumab, was given judiciously in a select number of patients. Corticosteroid use increased towards the end of our study, in July, when reports of its benefits among those on ventilatory support were released. It is important to note that remdesivir was not widely available in our institution during this study period. Convalescent plasma therapy and hemoperfusion were used as last-line therapeutic measures to control severe COVID-19 infections, usually in cytokine storm. Overall, use of these interventions was low in the ESKD population $(<10 \%)$, likely due to the patients' baseline kidney failure, which was usually a contraindication in most investigational trials.

With regards to supportive kidney replacement therapy, we were limited to use solely intermitted HD modality regardless of disease severity due to the limited machines available and limited manpower trained specifically for continuous renal replacement therapy.

The high in-hospital mortality rate of $25 \%$ in our center is similar to the mortality rate of $20-30 \%$ seen in larger study cohorts of ESKD patients who developed COVID19 in China, the United States of America, the United Kingdom and Spain. ${ }^{19-22}$ Our study population may be unique because the hospital is a referral center for 
moderate and severe cases of COVID-19. Patients may present later in the disease with more complications and increased risk for mortality. In fact, $18 \%$ of ESKD patients needed ICU admission during the course of their hospital stay with about $35 \%$ needing some form of respiratory support (17.65\% on high flow nasal cannula and $17.65 \%$ on mechanical ventilation).

Multivariable regression showed that risk for mortality increased with every unit decrease in PF ratio and need for invasive mechanical ventilation. Laboratory findings of increased white blood cell count and elevated inflammatory markers such as procalcitonin and LDH seemed to correlate with disease severity and in-hospital death but did not reach statistical significance in univariate and multivariate regression analysis. While these findings need to be confirmed in a larger study cohort of ESKD patients, they seem to be consistent with later reports involving a big study population..$^{23,24}$

During this study duration, protocols on the provision of HD among ESKD patients with COVID-19 varied per government-run or private institutions. All patients were screened for symptoms, and baseline temperature was checked before their dialysis session. General hygienic measures were enforced. Usually, if a patient presented with any symptom related to possible COVID-19, he or she was advised to seek consult at a nearest hospital or local health center for SARS-COV-2 nasopharyngeal RTPCR testing. This was because widespread testing was still limited during this time.

There remains to be a need for designated outpatient HD centers assigned for those with confirmed mild COVID-19 infection. At present, testing-based strategy is implemented in most centers, meaning ESKD patients with confirmed COVID-19 are advised hospital admission and are dialyzed and cohorted at inpatient HD units until they test negative for COVID-19 by PCR either once or twice depending on the requirements of their outpatient HD center. This becomes a challenge when hospital stay of asymptomatic or mildly-infected patients is unnecessarily prolonged due to persistent positive RTPCR results from prolonged viral shedding. ${ }^{25,26}$ Mean length of hospital stay in our study cohort was 28 days ( $2-82$ days) either due to complications of the disease or persistent positive RTPCR results. This post serious issues in terms of mitigating available resources such as hospital beds, dialysis supplies and healthcare workforce or staff, in an already resourceconstrained environment. A limited number of outpatient HD units accept ESKD patients with mild COVID-19 infection. Cohorting is usually done at the last shift, but this was at the prerogative of the dialysis center's medical director. On a larger, national scale, It would be prudent to form step-down units and designated outpatient HD centers for ESKD patients who are persistently positive by RTPCR but are otherwise asymptomatic and/or with mild COVID-19 infection.

This study is the first to describe the experience of ESKD patients in the Philippines hospitalized for COVID-19 infection. It highlights the unique challenges we face in resource-limited settings in terms of crisis management, supply and demand mismatches, and differences in therapeutic strategies. The limitations of the study include: (1) a relatively small sample size, (2) inability to assess out-of-hospital deaths, and (3) a relatively short follow-up period that precludes the ability to make any clear, generalizable associations. Lastly, other important aspects of the disease that are lacking but would have been important to note are: number of dialysis sessions missed due to COVID-19 infection and documentation of outpatient dialysis centers as a possible source of transmission. Information on the incidence of COVID-19 infection among ESKD patients in the outpatient setting and longterm outcomes also require further investigation.

\section{Conclusion}

ESKD patients on chronic HD are a vulnerable population at increased risk of mortality from COVID-19 infection. They present with similar clinical symptoms as the general population. The severity of acute respiratory failure, measured by low PF ratio on admission and need for invasive ventilatory support, are independently associated with inhospital mortality. Need for designated outpatient hemodialysis units for ESKD patients with COVID-19 and mitigating limited resources are some local challenges that remain to be addressed.

\section{Acknowledgment}

We would like to thank our dialysis nurses, internal medicine residents, nephrology fellows, and consultants for their hard work, commitment and excellent patient care throughout these trying times. We would also like to thank Dr. Emilio Villanueva III, MD, MSc, DPSP for his assistance with the presentation of this data.

\section{Disclosure}

The authors report no conflicts of interest for this work. 


\section{References}

1. Naicker S, Yang CW, Hwang SJ, Liu BC, Chen JH, Jha V. The novel coronavirus 2019 epidemic and kidneys. Kidney Int. 2020;1-5. doi:10.1016/j.kint.2020.03.001

2. Coronavirus disease (COVID-19) situation reports. Available from: https://www.who.int/emergencies/diseases/novel-coronavirus-2019/ situation-reports. Accessed August 15, 2020.

3. Cheng Y, Luo R, Wang $\mathrm{K}$, et al. Kidney disease is associated with in-hospital death of patients with COVID-19. Kidney Int. 2020;97 (5):829-838. doi:10.1016/j.kint.2020.03.005

4. Li J, Xu G. Lessons from the experience in Wuhan to reduce risk of COVID-19 infection in patients undergoing long-term hemodialysis. Clin J Am Soc Nephrol. 2020;15:717-719.

5. Clarke C, Prendecki M, Dhutia A, et al. High prevalence of asymptomatic COVID-19 infection in hemodialysis patients detected using serologic screening. J Am Soc Nephrol. 2020;31:1960-1975.

6. Prasad N, Bhatt M, Agarwal SK, et al. The adverse effect of COVID pandemic on the care of patients with kidney diseases in India. Kidney Int Reports. 2020;5(9):1545-1550. doi:10.1016/j. ekir.2020.06.034

7. United States Renal Data System. 2017 USRDS Annual Data Report: epidemiology of kidney disease in the United States. Am J Kidney Dis. 2018;71(3):S461-S500. doi:10.1053/j.ajkd.2018.01.024

8. Department of Health website. Available from: https://www.doh.gov. ph/2019-nCov. Accessed August 15, 2020.

9. PGH. Jose Rodriguez Memorial designated as exclusive COVID-19 hospitals - health dept. Available from: https://www.cnnphilippines. com/news/2020/3/20/Lung-Center-Jose-Rodriguez-Memorial-

Caloocan-COVID-exclusive-hospitals-DOH.html. Accessed August 15, 2020.

10. Zhou F, Yu T, Du R, et al. Clinical course and risk factors for mortality of adult inpatients with COVID-19 in Wuhan, China: a retrospective cohort study. Lancet. 2020;395(10229):1054-1062. doi:10.1016/S0140-6736(20)30566-3

11. Wu Z, McGoogan JM. Characteristics of and important lessons from the coronavirus disease 2019 (COVID-19) outbreak in China: summary of a report of 72314 cases from the Chinese center for disease control and prevention. JAMA. 2020;323(13):1239. doi:10.1001/ jama.2020.2648

12. Liang W, Guan W, Chen R, et al. Cancer patients in SARS-CoV-2 infection: a nationwide analysis in China. Lancet Oncol. 2020;21 (3):335-337. doi:10.1016/S1470-2045(20)30096-6

13. Lighter J, Phillips M, Hochman S, et al. Obesity in patients younger than 60 years is a risk factor for Covid-19 hospital admission. Clin Infect Dis. 2020;71(15):896-897. doi:10.1093/cid/ciaa415
14. Ma Y, Diao B, Lv X, et al. 2019 novel coronavirus disease in hemodialysis (HD) patients: report from one HD center in Wuhan, China. medRxiv. 2020. doi:10.1101/2020.02.24.20027201

15. Trivedi M, Shingada A, Shah M, Khanna U, Karnik ND, Ramachandran R. Impact of COVID-19 on maintenance haemodialysis patients: the Indian scenario. Nephrology. 2020;25(12):929-932. doi:10.1111/nep.13760

16. Ferrey AJ, Choi G, Hanna RM, et al. A case of novel coronavirus disease 19 in a chronic hemodialysis patient presenting with gastroenteritis and developing severe pulmonary disease. Am J Nephrol. 2020;51(5):337-342. doi:10.1159/000507417

17. Henry BM, De Oliveira MHS, Benoit S, Plebani M, Lippi G. Hematologic, biochemical and immune biomarker abnormalities associated with severe illness and mortality in coronavirus disease 2019 (COVID-19): a meta-analysis. Clin Chem Lab Med. 2020;58 (7):1021-1028. doi:10.1515/cclm-2020-0369

18. Li X, Xu S, Yu M, et al. Risk factors for severity and mortality in adult COVID-19 inpatients in Wuhan. J Allergy Clin Immunol. 2020;146(1):110-118. doi:10.1016/j.jaci.2020.04.006

19. Xiong F, Tang H, Liu L, et al. Clinical characteristics of and medical interventions for COVID-19 in hemodialysis patients in Wuhan, China. J Am Soc Nephrol. 2020;31(7):1387-1397. doi:10.1681/ ASN.2020030354

20. Valeri AM, Robbins-Juarez SY, Stevens JS, et al. Presentation and outcomes of patients with ESKD and COVID-19. J Am Soc Nephrol. 2020;31(7):1409-1415. doi:10.1681/ASN.2020040470

21. Fisher M, Yunes M, Mokrzycki MH, Golestaneh L, Alahiri E, Coco M. Chronic hemodialysis patients hospitalized with COVID-19 - short-term outcomes in Bronx, New York. Kidney360. 2020;1(8):755-762. doi:10.34067/kid.0003672020

22. Goicoechea M, Sánchez Cámara LA, Macías N, et al. COVID-19: clinical course and outcomes of 36 hemodialysis patients in Spain. Kidney Int. 2020;98(1):27-34. doi:10.1016/j.kint.2020.04.031

23. Santus P, Radovanovic D, Saderi L, et al. Severity of respiratory failure at admission and in-hospital mortality in patients with COVID-19: a prospective observational multicentre study. $B M J$ Open. 2020;10(10):e043651. doi:10.1136/bmjopen-2020-043651

24. Rosenthal N, Cao Z, Gundrum J, Sianis J, Safo S. Risk factors associated with in-hospital mortality in a US national sample of patients with COVID-19. JAMA Netw Open. 2020;3(12):e2029058. doi:10.1001/jamanetworkopen.2020.29058

25. Kwan BCH, Leung CB, Szeto CC, et al. Severe acute respiratory syndrome in dialysis patients. $J$ Am Soc Nephrol. 2004;15 (7):1883-1888. doi:10.1097/01.ASN.0000131522.16404.1F

26. Liu W-D, Chang S-Y, Wang J-T, et al. Prolonged virus shedding even after seroconversion in a patient with COVID-19. J Infect. 2020;81 (2):318-356. doi:10.1016/j.jinf.2020.03.063
The International Journal of Nephrology and Renovascular Disease is an international, peer-reviewed open-access journal focusing on the pathophysiology of the kidney and vascular supply. Epidemiology, screening, diagnosis, and treatment interventions are covered as well as basic science, biochemical and immunological studies. The manuscript management system is completely online and includes a very quick and fair peer-review system, which is all easy to use. Visit http://www.dovepress.com/testimonials.php to read real quotes from published authors. 